\title{
A photometric study of BH Cas`
}

\author{
S. Zoła ${ }^{1,2}$, P. Niarchos ${ }^{3}$, V. Manimanis ${ }^{3}$, and A. Dapergolas ${ }^{4}$ \\ 1 Astronomical Observatory of the Jagiellonian University, ul. Orla 171, 30-244 Cracow, Poland \\ 2 Mt. Suhora Observatory of the Pedagogical University, ul. Podchorążych 2, 30-084 Cracow, Poland \\ 3 Department of Astrophysics, Astronomy and Mechanics, Faculty of Physics, University of Athens, \\ Panepistimiopolis, 15784 Zografos, Athens, Greece \\ ${ }^{4}$ Institute of Astronomy and Astrophysics, National Observatory of Athens, 15236, Palaia Penteli, Athens, Greece
}

Received 4 April 2000 / Accepted 8 May 2001

\begin{abstract}
New CCD observations of the contact binary system BH Cas are presented in this paper. New times of minima and an ephemeris based on our observations are also given. Complete light curves obtained in the $V, R$ and $I$ filters have been analyzed with the Wilson-Devinney code to derive the geometrical and physical parameters of the system. The final solution leads to a contact configuration $(f \approx 21-22 \%)$. We have found that the photometric mass ratio differs from the spectroscopic one by about $13 \%$, a discrepancy which is not uncommon for other W UMa systems. The absolute elements of the system are used to study its evolutionary status. The results show that BH Cas is a fairly evolved W-type W UMa system.
\end{abstract}

Key words. binaries: eclipsing BH Cas

\section{Introduction}

BH Cas $\left(V=12.58^{\mathrm{m}}, R=12.32^{\mathrm{m}}, I=11.67^{\mathrm{m}}, \mathrm{RA}=\right.$ $00^{\mathrm{h}} 21^{\mathrm{m}} 21^{\mathrm{s}} 4$, DEC $\left.=+59^{\circ} 09^{\prime} 05^{\prime \prime} \cdot 2(\mathrm{~J} 2000)\right)$, was discovered as a variable star by Beljawsky in 1928 (Beljawsky 1931), who did not list its period, nor its maximum or minimum magnitudes. Until recently, the only other observations of this system were made by Kukarkin (1938), who tentatively classified BH Cas as a W UMa-type system with a period near $0.5^{\mathrm{d}}$ and an amplitude of $0.4 \mathrm{mag}$. Because Ahnert \& Hoffmeister (1943) could not find BH Cas as a variable star (after searching for it both on photographic plates and visually) there was a complete absence of additional efforts to observe the system for the next 50 years.

In 1994, Metcalfe (1994) took CCD images on one night using the Steward Observatory $0.91 \mathrm{~m}$ telescope on Kitt Peak that clearly showed the variability of $\mathrm{BH}$ Cas. In the following years he used the $1.5 \mathrm{~m}$ telescope for 12 nights of CCD photometry, obtaining a complete light curve of the binary in $V$ and partial curves in $R$ and $I$, as well as the $2.1 \mathrm{~m}$ telescope at McDonald Observatory

Send offprint requests to: S. Zoła,

e-mail: sfzola@cyf-kr.edu.pl

* Based on observations collected at the Kryonerion Observatory, Greece. The original observations are available in electronic form at the CDS via anonymous ftp to cdsarc.u-strasbg.fr (130.79.128.5) or via

http://cdsweb.u-strasbg.fr/cgi-bin/qcat?J/A+A/374/164 for 10 nights of photoelectric photometry, obtaining complete light curves in $B$ and $U$. Finally, he used the echelle spectrograph on the $2.1 \mathrm{~m}$ telescope in order to obtain radial velocities for the system (Metcalfe 1999). However, in his photometry reduction procedure he made the assumption that the corrected maxima in the $U$ light curve were equal, thus sacrificing the measurement of a possible O' Connell effect and the subsequent study of possible spots. Metcalfe used a genetic-algorithm based method to optimally fit the observed $U B V$ curves with a model produced by the Wilson-Devinney code.

The purpose of this study is to present new, high quality observations extended to the near infrared bands $R$ and $I$, and to check if there is spot activity in the system. Using our data without any corrections or assumptions regarding the presence of possible O'Connell effect, we found no such effect or other noticeable irregularities in the light curves, so that we performed light curve modelling without any spot. Two models, one with the mass ratio fixed at the value derived from spectroscopy and a second model with the mass ratio adjusted are presented in Sect. 3 .

\section{New photometric data}

\subsection{Observations}

The system was observed for two consecutive nights on October 15 and 16, 1998. The instruments used were the $1.22 \mathrm{~m}$ Cassegrain reflector at the Kryonerion 


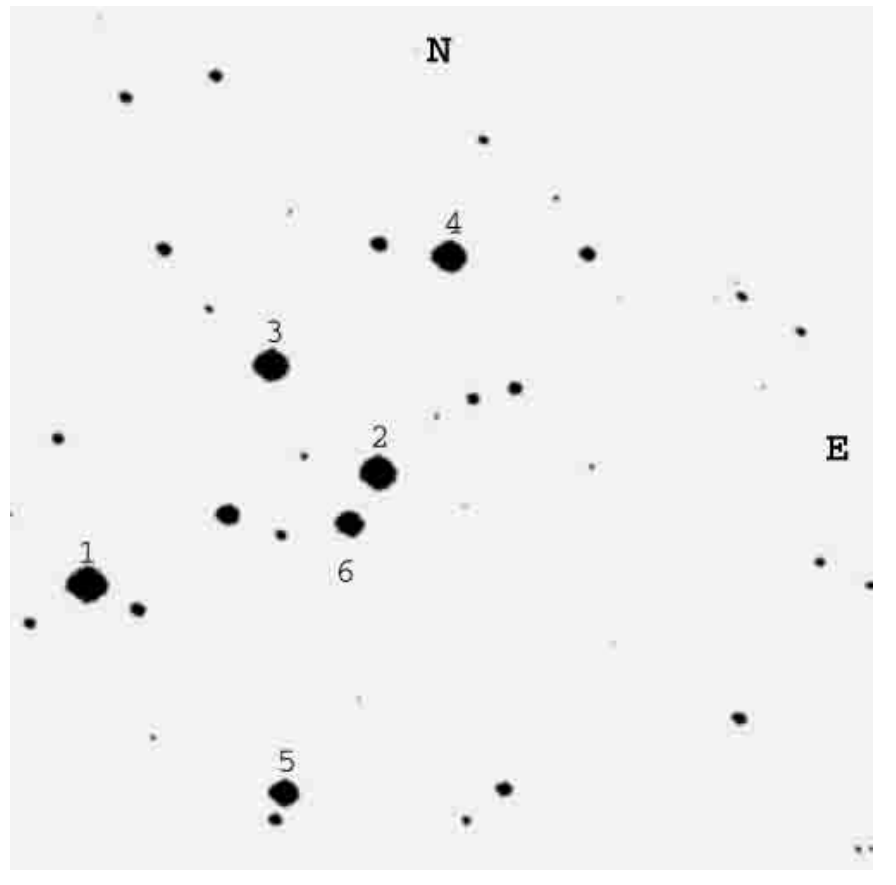

Fig. 1. $2^{\prime} \times 2^{\prime}$ frame taken with the Kryonerion CCD camera on the position of BH Cas through the I filter. Star No. 2 is the target star, while No. 6 is the comparison star.

Astronomical Station of the National Observatory of Athens, Greece, and a CCD camera. The Photometrics CCD camera uses a Class I, SI502 CCD chip, cooled by a three stage Peltier element and traditional chilled water cooling that provides a stable CCD working temperature of -40 degrees Celsius. The CCD chip has $516 \times 516$ useful pixels of $24 \times 24$ microns. The chip's readout is done at $40 \mathrm{khz}$ with a 16-bit A/D converter. The measured gain corresponds to 5.17 electrons/ADU with a readout noise of 9.0 electrons rms (1.74 ADU). Under this configuration the dark current is 1.03 electrons/pixel/second at a measured temperature of -40 degrees Celsius. The CCD camera is equipped with a set of $U, B, V, R$ and $I$ Bessell filters (size of each filter $5 \mathrm{~cm} \times 5 \mathrm{~cm}$ ) and it is attached at the Cassegrain focus of the $1.22 \mathrm{~m}$ telescope at the Kryonerion Astronomical Station. The scale on the chip is $0.30 \mathrm{arcsec} / \mathrm{pixel}$. A detailed description of the CCD chip was given by Sinachopoulos et al. (1998). BH Cas was observed in $V, R$ and $I$ filters. A total number of 215, 215 and 216 frames were obtained in $V, R$ and $I$, respectively.

\subsection{Data reduction}

The frames have been reduced for bias, dark and flat using the MIDAS package. In order to obtain differential magnitudes, aperture photometry with the DAOPHOT II program (Stetson 1987) was employed. We derived instrumental magnitudes of the target star along with the six brightest visible stars on all frames. After checking the colours of BH Cas and all reduced stars, we have chosen the nearest, about 1 mag fainter than BH Cas, as our comparison star (star No. 6 in Fig. 1). The colour difference ( $V-R$ and $R-I$ ), between the target star and our comparison star was the smallest (less than $0.05 \mathrm{mag}$ ). We have also checked its constancy against all reduced stars. We found no variability larger than 0.01 mag during two nights. The light curve of BH Cas in $V, R$ and $I$ filters is shown in Figs. 3 and 4 (points) in flux units, normalized to about 1.0 at phase 0.25 .

\section{3. $O-C$ analysis}

Metcalfe (1999) noticed no significant changes of the period of $\mathrm{BH}$ Cas when analyzing the times of the primary minima and he determined the following linear ephemeris for this system:

Min I = HJD 2449998.6187 + 0.40589004 E.

For the O-C study, BH Cas was also observed at the Mt. Suhora observatory with a two-channel photometer. A description of the instrument was given by Kreiner et al. (1993). From the $V$ and $R$ observations, made around the predicted times of the primary and secondary minima, we derived several new times of minima. From the CCD observations at the Kryonerion observatory we determined one new time of the primary minimum and two times of the secondary minimum. Additionally, we observed $\mathrm{BH}$ Cas at the Kryonerion observatory near the predicted times of minima, in November 1999 and November 2000. From these observations we obtained five new times for both primary and secondary minima. Two (one primary and one secondary) minima of $\mathrm{BH}$ Cas were observed at the Astronomical Observatory of the Jagiellonian University using a $50 \mathrm{~cm}$ telescope and a CCD camera in October 2000 and two more at the Skibotn Astronomical Observatory with a Tromso CCD photometer and a $50 \mathrm{~cm}$ telescope in January 2001. For the determination of the new times of minima we employed the Kwee-van Woerden (1956) method. Based on this enlarged time span, we determined new linear and also parabolic elements. For this purpose we used only the times of minima observed either photoelectrically or with a CCD camera, 39 of them altogether. The seventh time of minimum listed in Table 1 was not used due to its much lower accuracy. The new linear elements are:

Min I = HJD 2449998.6183(3) + 0.40589160(5) E.

The parabolic elements are:

Min I = HJD 2449998.6187(3)+0.4058907(2) E $+0.52(15) \times 1.0^{-10} E^{2}$.

All new times of minima along with the $\mathrm{O}-\mathrm{C}$ values for both cases are given in Table 1 and the respective diagram is shown in Fig. 2. The upper panel shows the differences between observed and computed times of minima calculated with the parabolic elements while the bottom panel with the linear elements. O-C's of the most recent times 
Table 1. New times of minima derived from photoelectric and CCD observations.

\begin{tabular}{cllrrccl}
\hline No & HJD & Error & $(\mathrm{O}-\mathrm{C})_{1}$ & $(\mathrm{O}-\mathrm{C})_{2}$ & Type & Filter & Remarks \\
\hline 1 & 2450694.5183 & 0.0004 & -0.00112 & -0.00060 & sec & $R$ & pe \\
2 & 2450694.5210 & 0.0004 & 0.00157 & 0.00209 & sec & $V$ & pe \\
3 & 2450744.6458 & 0.0011 & -0.00123 & -0.00070 & pri & $R$ & pe \\
4 & 2450744.6466 & 0.0012 & -0.00043 & 0.00009 & pri & $V$ & pe \\
5 & 2450757.4297 & 0.0006 & -0.00291 & -0.00238 & sec & $R$ & pe \\
6 & 2450757.6358 & 0.0005 & 0.00023 & 0.00076 & pri & $V$ & pe \\
7 & 2450758.449 & 0.020 & - & - & pri & $V$ & pe: \\
8 & 2450837.3957 & 0.0006 & 0.00243 & 0.00298 & sec & $V$ & pe \\
9 & 2450837.3959 & 0.0003 & 0.00263 & 0.00318 & sec & $R$ & pe \\
10 & 2450838.4054 & 0.0006 & -0.00259 & -0.00204 & pri & $V$ & pe \\
11 & 2450838.4065 & 0.0003 & -0.00149 & -0.00094 & pri & $R$ & pe \\
12 & 2451102.4396 & 0.0003 & -0.00087 & -0.00040 & sec & $R$ & CCD \\
13 & 2451102.4402 & 0.0002 & -0.00027 & 0.00019 & sec & $V$ & CCD \\
14 & 2451102.4404 & 0.0002 & -0.00007 & 0.00039 & sec & $I$ & CCD \\
15 & 2451103.2514 & 0.0003 & -0.00086 & -0.00039 & sec & $R$ & CCD \\
16 & 2451103.2516 & 0.0004 & -0.00066 & -0.00019 & sec & $I$ & CCD \\
17 & 2451103.4547 & 0.0030 & -0.00050 & -0.00003 & pri & $V$ & CCD \\
18 & 2451103.4549 & 0.0028 & -0.00030 & 0.00016 & pri & $R$ & CCD \\
19 & 2451103.4554 & 0.0028 & 0.00019 & 0.00066 & pri & $I$ & CCD \\
20 & 2451489.4583 & 0.0003 & 0.00008 & 0.00012 & pri & $V$ & CCD \\
21 & 2451839.3373 & 0.0009 & 0.00062 & -0.00004 & pri & $R$ & CCD \\
22 & 2451840.3513 & 0.0005 & -0.00010 & -0.00077 & sec & $R$ & CCD \\
23 & 2451850.4994 & 0.0007 & 0.00070 & 0.00000 & sec & $I$ & CCD \\
24 & 2451851.5137 & 0.0004 & 0.00027 & -0.00042 & pri & $I$ & CCD \\
25 & 2451852.5293 & 0.0007 & 0.00114 & 0.00044 & sec & $I$ & CCD \\
26 & 2451915.2388 & 0.0009 & 0.00039 & -0.00046 & pri & $R$ & CCD \\
27 & 2451920.3137 & 0.0007 & 0.00164 & 0.00077 & sec & $I$ & CCD \\
\hline & & & & & & &
\end{tabular}

$(\mathrm{O}-\mathrm{C})_{1}$-from parabolic fit.

$(\mathrm{O}-\mathrm{C})_{2}$-from linear fit.

of minima (October and November 2000) calculated with linear elements are systematically positive, while those obtained with parabolic elements are closer to 0 . The quality of the fits as described by $\chi^{2}$ are: 1.7841 and 1.4778 , for the linear and parabolic elements respectively. To calculate phases in this paper, we used the period from the new linear ephemeris and the time of primary minimum $\mathrm{JD}_{\text {hel }}=2451103.4550$.

\section{Light curve modelling}

\subsection{Solution with the spectroscopic mass ratio}

As can be seen from the light curve, there is almost no O'Connell effect visible, but there is a small asymmetry just before the start of the primary eclipse. Thus we limited our search for the best fit only to models without any spot on the components. We used the WilsonDevinney (W-D) code, ver. 1996 (Wilson \& Devinney 1971; Wilson 1993) with the supplied differential correction search method. Obtaining a unique fit could be a very difficult task, if we had no constraints on the crucial parameters of a binary system. Lines from both components are observed in the spectrum of BH Cas and a double-lined radial velocity curve was published by Metcalfe (1999).
In this paper we follow Metcalfe's definition for the primary (more massive) and the secondary (hotter, less massive) component. Therefore the subscripts 1 and 2 refer to the primary and secondary component, respectively. Such a naming convention required the introduction of a phase shift of the order of $0.5 \mathrm{P}$ to have an agreement between the synthetic light curve produced by the W-D code and observations. We fixed the mass ratio $q$ at Metcalfe's value 0.475 in our computations. Metcalfe's determination of the primary star temperature based on BH Cas colour resulted in $4600 \mathrm{~K}$, but he finally adopted $5000 \mathrm{~K}$, derived from light curve modelling. Our preliminary computations with the new $V R I$ data also indicated that a better fit could be obtained for a somewhat higher temperature. We decided to estimate the temperature independently from the light curve modelling. A classification spectrum of $\mathrm{BH}$ Cas was taken at the David Dunlap Observatory with the $1.8 \mathrm{~m}$ telescope. A description of the instrument was published by Lu \& Rucinski (1999). On the basis of this single spectrum it turned out that the spectral type of BH Cas is F8 $( \pm 2)$ (Rucinski \& Lu 2000, private communication). We made no attempt to derive temperatures of both components from this, composite spectrum. Since the observed light curves could be fitted very well by very large range of temperatures, for further analysis we set the hotter 


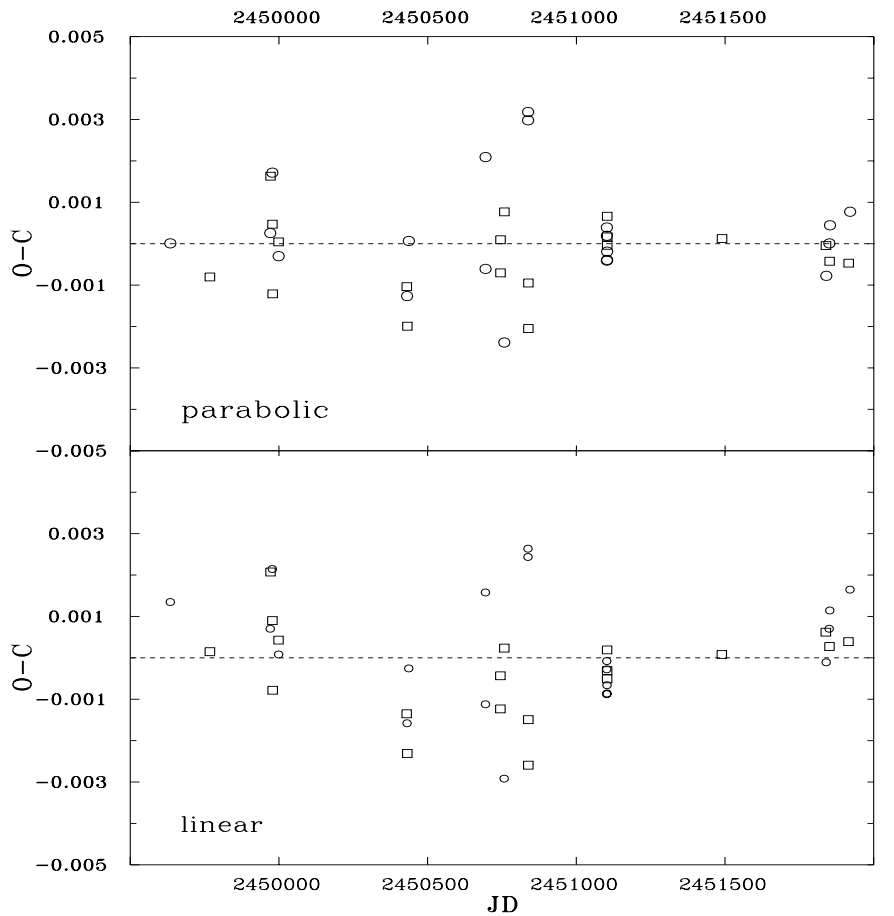

Fig. 2. The $\mathrm{O}-\mathrm{C}$ diagrams for $\mathrm{BH}$ Cas. The upper pannel shows $\mathrm{O}-\mathrm{C}$ calculated with the parabolic elements, the bottom pannel with linear elements. Primary minima are denoted by squares, while the secondary ones by circles.

component's temperature to $6000 \mathrm{~K}$ as corresponding to the spectral type from the calibration published by Harmanec (1988).

With the secondary star's temperature and the mass ratio fixed, we proceeded with the differential correction search, trying to find the best solution simultaneously in the $V, R$ and $I$ filters. During these computations, we additionally fixed the albedo and gravity darkening coefficients at their theoretical values 0.5 and 0.32 , respectively. The limb darkening coefficients were taken from DíazCordovés et al. (1995) and Claret et al. (1995), respective to the effective wavelength of each filter and temperatures of components. We assumed that there is no third light in the system. The following parameters have been treated as free: the inclination, the temperature of the primary star, the potentials of the components, and the primary component's luminosity. The computation was stopped when the probable errors were larger than the applied corrections. Eventually, a contact configuration was obtained. The results are given in Table 2. Theoretical light curves corresponding to this solution (lines) are plotted in Figs. 3 and 4, along with observations (squares). As one can see, the fit is almost perfect, with only small departures just before the primary minimum. In the upper panel of Fig. 4 we have also plotted a theoretical light curve in the $U$ filter, against the observed curve for which the data was taken from Metcalfe (1999). The fit in the $U$ region is also very good. In order to obtain the theoretical light curve in the $U$ filter, we adjusted only the primary star's
Table 2. Results derived from the light curve modelling.

\begin{tabular}{|c|c|c|}
\hline parameter & $q$ fixed & $q$ adjusted \\
\hline phase shift & $0.4994 \pm 0.0004$ & $0.4993 \pm 0.0004$ \\
\hline$i$ (degrees) & $71.20 \pm 0.13$ & $71.66 \pm 0.16$ \\
\hline$T_{1}(\mathrm{~K})$ & $5550 \pm 22$ & $5555 \pm 22$ \\
\hline$T_{2}(\mathrm{~K})$ & $* 6000$ & $* 6000$ \\
\hline$\Omega_{1}$ & $2.769 \pm 0.004$ & $2.645 \pm 0.009$ \\
\hline$\Omega_{2}$ & $* * 2.769$ & $* * 2.645$ \\
\hline$q\left(=m_{2} / m_{1}\right)$ & $* 0.475$ & $0.411 \pm 0.007$ \\
\hline$L_{1}(V)$ & $6.793 \pm 0.033$ & $7.184 \pm 0.042$ \\
\hline$L_{1}(R)$ & $6.957 \pm 0.028$ & $7.342 \pm 0.040$ \\
\hline$L_{1}(I)$ & $7.105 \pm 0.024$ & $7.479 \pm 0.037$ \\
\hline$L_{2}(V)$ & $* * 4.8865$ & $* * 4.3043$ \\
\hline$L_{2}(R)$ & $* * 4.7717$ & $* * 4.1975$ \\
\hline$L_{2}(I)$ & $* * 4.6195$ & $* * 4.0074$ \\
\hline$x_{1}=x_{2}(V)$ & $* 0.680$ & $* 0.679$ \\
\hline$x_{1}=x_{2}(R)$ & $* 0.593$ & $* 0.593$ \\
\hline$x_{1}=x_{2}(I)$ & $* 0.491$ & $* 0.490$ \\
\hline$r_{1}$ pole & $0.4287 \pm 0.0007$ & $0.4409 \pm 0.0010$ \\
\hline$r_{2}$ pole & $0.3064 \pm 0.0009$ & $0.2959 \pm 0.0013$ \\
\hline$r_{1}$ side & $0.4582 \pm 0.0010$ & $0.4729 \pm 0.0015$ \\
\hline$r_{2}$ side & $0.3213 \pm 0.0007$ & $0.3102 \pm 0.0037$ \\
\hline$r_{1}$ back & $0.4901 \pm 0.0009$ & $0.5040 \pm 0.0046$ \\
\hline$r_{2}$ back & $0.3614 \pm 0.0013$ & $0.3513 \pm 0.0083$ \\
\hline$L_{1} /\left(L_{1}+L_{2}\right)(V)$ & $0.582 \pm 0.004$ & $0.625 \pm 0.007$ \\
\hline$L_{1} /\left(L_{1}+L_{2}\right)(R)$ & $0.593 \pm 0.004$ & $0.636 \pm 0.007$ \\
\hline$L_{1} /\left(L_{1}+L_{2}\right)(I)$ & $0.606 \pm 0.004$ & $0.625 \pm 0.004$ \\
\hline$\%$ overcontact & $21 \%$ & $22 \%$ \\
\hline$S U M(W * R E S * * 2)$ & 0.01628 & 0.01589 \\
\hline
\end{tabular}

* - not adjusted, $\quad * *$ - computed.

$L_{1}, L_{2}$ : W-D program input values.

luminosity and the phase shift, while the other parameters were fixed at the values resulting from our best fit.

\subsection{Search for the photometric mass ratio}

Our final attempt was to determine the mass ratio photometrically, for two reasons. First, Metcalfe (1999) posed some questions regarding the accuracy of the mass determination of the two components. He admits that there are some possibilities that could have led to biased estimates of the masses of $\mathrm{BH}$ Cas, e.g. the derivation of the radial velocities exclusively from the $\mathrm{H}_{\alpha}$ line, considerable scatter in the observations and assumed Gaussian profiles for the correlation peaks. Second, there were questions about the possible existence of a systematic trend between the mass ratios derived photometrically and spectroscopically.

To find the best fit, we again used the W-D code with the differential corrections search method and let the $q$ parameter be free. Additionally, we set the starting 

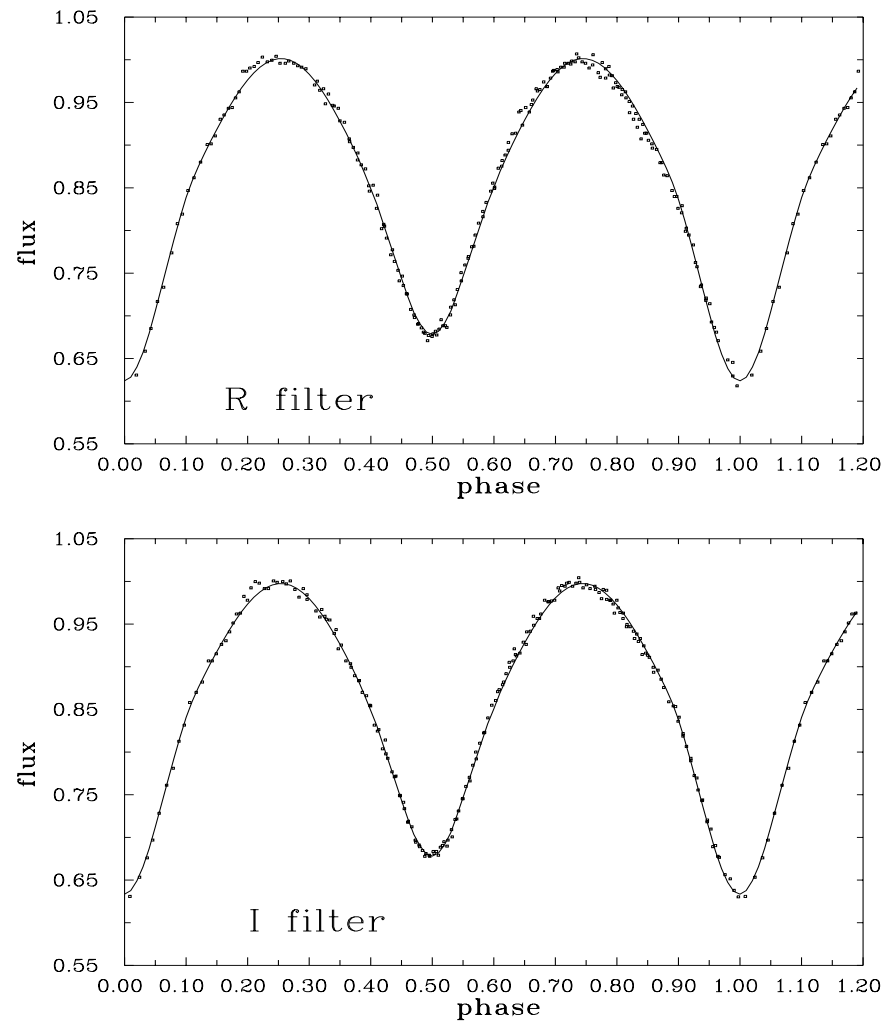

Fig. 3. Comparison between theoretical and observed light curves of $\mathrm{BH}$ Cas ( $R$ and $I$ filters). Individual observations are shown by squares and theoretical curves by lines.

parameters for this search to the results derived from the solution with the mass ratio fixed. The computations converged again to a contact configuration with the photometric mass ratio somewhat smaller than the spectroscopic one. The results are given in Table 2 . We tried to plot the theoretical light curves in Figs. 3 and 4, but they were practically undistinguishable from those obtained in the solution with the mass ratio fixed. Please note that all errors listed in Table 2 are standard errors.

It is not surprising that the derived photometric mass ratio $q_{\mathrm{ptm}}$ differ from the spectroscopic one $q_{\mathrm{sp}}$. Discrepancies between $q_{\mathrm{sp}}$ and $q_{\mathrm{ptm}}$ have been noticed since the first determinations of photometric mass ratios for contact binaries (Mochnacki \& Doughty 1972a,b; Wilson \& Devinney 1973). It seems that such discrepancies also exist for other W UMa systems (Maceroni \& Van't Veer 1996), in which the photometric mass ratio is determined with high accuracy. This is the case for totally eclipsing systems, while a larger difference between spectroscopic and photometric mass ratio is found usually for binaries undergoing partial eclipses (Wilson 1978).

An attempt to reconcile $q_{\mathrm{sp}}$ and $q_{\mathrm{ptm}}$ by a proper simultaneous light and velocity solution has been made by Wilson (1979) and Van Hamme \& Wilson (1985) in the case of AE Phe. Nevertheless, they obtained $q_{\mathrm{sp}} \neq q_{\mathrm{ptm}}$. It seems that the simultaneous solution does not really help in understanding the overall situation and very probably
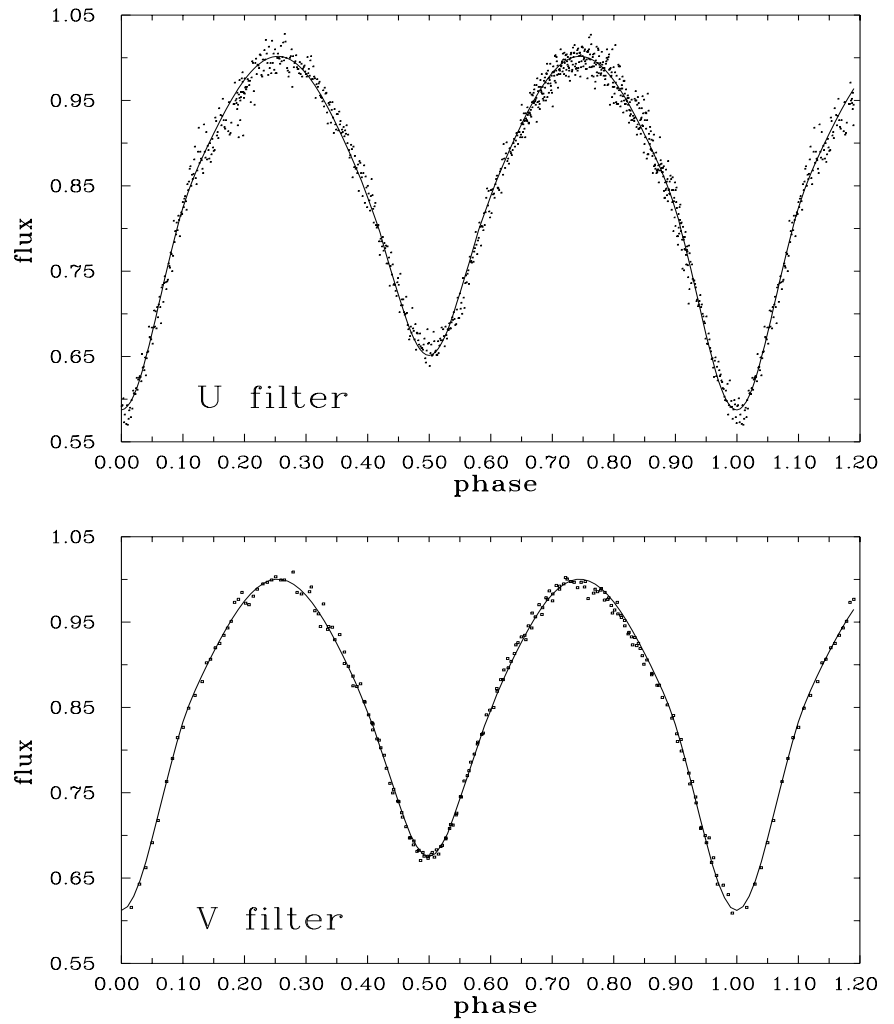

Fig. 4. Comparison between theoretical and observed light curves of $\mathrm{BH}$ Cas ( $U$ and $V$ filters). Individual observations are shown by squares and theoretical curves by lines.

systematic effects affect the radial velocities and the spectroscopic mass ratios. A hint towards the solution of the $q$-discrepancy was given by Niarchos \& Duerbeck (1991). If the discrepancy is plotted as $\Delta q / q=2\left(q_{\mathrm{sp}}-q_{\mathrm{ptm}}\right) /\left(q_{\mathrm{sp}}+\right.$ $\left.q_{\mathrm{ptm}}\right)$ for systems with total eclipses as a function of spectral type, it is noted that $q_{\mathrm{sp}}$ is systematically larger than $q_{\mathrm{ptm}}$ for early spectral types (A, F), while it is smaller for types $\mathrm{G}$ and $\mathrm{K}$. In the case of $\mathrm{BH}$ Cas, its position on the above diagram confirms the validity of the above hypothesis.

\section{Discussion}

New, accurate measurements of the contact binary star BH Cas have been acquired in the $V, R$ and $I$ filters. We have chosen a comparison star with colours very close to those of the target star, thus minimizing the effects of the colour extinction term. Our light curve shows no significant O'Connell effect. Only a small asymmetry just before the primary minimum can be noticed. The light curve solution was performed by assuming that there are no spots on the surfaces of the two components, as it is clear from the shape of the light curves. The case of $\mathrm{BH}$ Cas is rather an exception, since most of the light curves of W-type W UMa systems show asymmetries and irregularities usually interpreted by invoking cool (and/or hot) spots on the surfaces of the two components. 
Table 3. Absolute parameters of BH Cas.

\begin{tabular}{rrrrrr}
\hline $\mathcal{M}_{\mathrm{p}} / \mathcal{M}_{\odot}$ & $\mathcal{M}_{\mathrm{s}} / \mathcal{M}_{\odot}$ & $R_{\mathrm{p}} / R_{\odot}$ & $R_{\mathrm{s}} / R_{\odot}$ & $L_{\mathrm{p}} / L_{\odot}$ & $L_{\mathrm{s}} / L_{\odot}$ \\
\hline 0.73 & 0.35 & 1.09 & 0.78 & 1.01 & 0.72 \\
\pm 0.15 & \pm 0.07 & \pm 0.05 & \pm 0.06 & \pm 0.07 & \pm 0.09 \\
\hline
\end{tabular}

An extensive search for the best fit to the observed light curves was performed with a new temperature of the hotter component derived from a classification spectrum of BH Cas. This temperature, corresponding to the spectral type $\mathrm{F} 8 \pm 2$, is higher by more than $1000 \mathrm{~K}$ than that inferred by Metcalfe (1999) on the basis of BH Cas colours and used in his light curve modelling. We consider the temperature derived from spectroscopy as being more reliable, especially because the colours derived by Metcalfe: $(U-B)=0.04,(B-V)=0.37,(V-I)=0.91$ and $(R-I)=0.65$ (Metcalfe 2001, private communication) are neither consistent with colours of a K4 nor of a F8 star. The above colours were not dereddened due to problems with establishing a reliable zero-point for the B magnitude (Metcalfe 2001, private communication). Let us consider the difference between the observed $(V-I)$ and $(R-I)$ colours of BH Cas and normal dwarfs with temperatures of $5000 \mathrm{~K}$ and $6000 \mathrm{~K}$ taken from the calibration by Bessell (1979). For a $5000 \mathrm{~K}$ dwarf we have: $\Delta(V-I)=-0.02, \Delta(R-I)=+0.22$, while for a $6000 \mathrm{~K}$ $\operatorname{star} \Delta(V-I)=+0.29$ and $\Delta(R-I)=+0.35$. As one can see, if BH Cas was as cool as $5000 \mathrm{~K}$, its $(R-I)$ colour would have been reddened by more than 0.2 mag while $(V-I)$ is not reddened at all. Unless there is an additional hot source in BH Cas (i.e. a third star) we consider this as an additional argument for the components of BH Cas having higher temperatures than these inferred from the $(V-I)$ and $(R-I)$ colours alone. Taking into account the luminosities from Table 3 the distance to BH Cas can be estimated. From the computed absolute magnitude $M_{V}=4.22$ of the system and its visual apparent magnitude $m_{V}=12.58$, and assuming no interstellar reddening, the distance is found to be about $d=470 \mathrm{pc}$ (the upper limit), while $d=390 \mathrm{pc}$, if an average reddening (1 mag/kpc) is assumed.

We present two solutions: the first with the spectroscopic value of $q=0.475$, derived by Metcalfe (1999) from his radial velocity curves, and the second one with the mass ratio being adjusted. We found that there is some discrepancy between the mass ratio derived on the basis of radial velocity curves and that obtained from photometry alone. Such a discrepancy also exists for other W UMa systems (Maceroni \& Van't Veer 1996) and it was shown by Niarchos \& Duerbeck $(1991,1997)$ that this discrepancy changes as a function of the mean effective temperature (or the spectral type). The tendency is clearer for totally eclipsing systems and the results for BH Cas confirm it.

By using the parameters derived from the present photometric study and the spectroscopic elements given by Metcalfe (1999), we computed the absolute parameters of the system given in Table 3 . The mass ratio of $\mathrm{BH}$

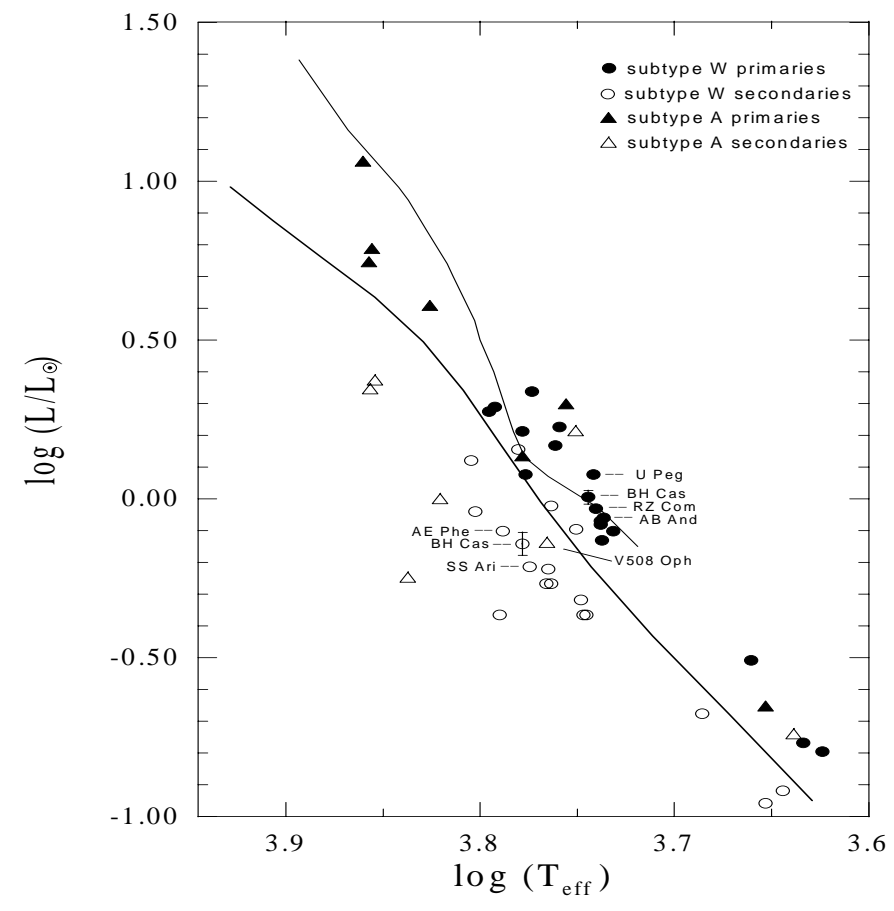

Fig. 5. Locations of the primary and secondary components of BH Cas along with other $24 \mathrm{~W}$ UMa systems in the H-R diagram. Systems that are closest to BH Cas are individually identified by their variable star designations. Also shown are the ZAMS and TAMS lines from Vandenberg (1985) for stars of solar metallicity. Error bars are given only for the components of BH Cas (but they are typical of the whole sample).

Cas is near the average for W-type W UMa systems, but the individual masses are near the low end of typical values. The mass of the secondary component is particularly low, but this is not uncommon among W UMa systems (see, for example, the table with absolute elements of W UMa systems in Maceroni \& van't Veer 1996). The computed absolute elements of BH Cas are used to estimate the evolutionary status of the system by means of the mass-radius (MR), mass-luminosity (ML) and HR diagrams of Hilditch et al. (1988), who showed that the evolution-sensitive MR diagram should be considered as the principal indicator of the evolutionary state of the individual stars. In the first two diagrams the primary component of BH Cas lies slightly beyond the TerminalAge Main Sequence (TAMS), where the primaries of some other W-type W UMa systems are found. It seems that the primary component of BH Cas is oversized and overluminous for its ZAMS mass. The position of the secondary component in the same diagrams is among the secondaries of other W-type W UMa systems, but again it has larger radius and luminosity than expected for its ZAMS mass. The situation is slightly different if we use the H-R diagram. In Fig. 5 the locations of the primary and secondary components of BH Cas along with other 24 W UMa systems (selected from Table 3 of Maceroni \& van't Veer 1996 on the base of the quality of their photometric and spectroscopic data) are depicted. The primary component of 
BH Cas falls on the TAMS and it is very close to the primaries of the W-type systems AB And, RZ Com and U Peg, while the location of the secondary is to the left of the main-sequence band (very close to the secondaries of $\mathrm{AE}$ Phe, SS Ari and U Peg), as a result of luminosity transfer from the primary to secondary (Hilditch et al. 1988). The previous analysis suggests that $\mathrm{BH}$ Cas is a fairly evolved W-type W UMa system.

W UMa binaries are known as one of the strong coronal X-ray emission sources. The X-ray luminosities measured from each of these systems are in the range $10^{29}-10^{30} \mathrm{erg} \mathrm{s}^{-1}$, which are $10^{2}-10^{3}$ times higher than that of a typical solar flare (Choi \& Dotani 1998). Such high X-ray luminosities of this type of binary systems are believed to be related to a high level of magnetic activity, caused by dynamo action (Maceroni \& van't Veer 1996; Choi \& Dotani 1998). According to Priest, Parnell \& Martin (1994) the mechanism for X-ray production is caused by reconnection of the emerging flux with an overlying coronal magnetic field.

Brandt et al. (1997) reported the first X-ray detection of BH Cas, which was serendipitously observed during X-ray observations of the Local Group galaxy IC 10. They derived an X-ray flux of $4.2 \times 10^{-14} \mathrm{erg} \mathrm{cm}^{-2} \mathrm{~s}^{-1}$. If we adopt as an upper limit on the distance of $\mathrm{BH}$ Cas the value $470 \mathrm{pc}$, then the X-ray luminosity appears to be less than about $1.1 \times 10^{30} \mathrm{erg} \mathrm{s}^{-1}$.

Emission of X-rays from other W UMa systems was also found and reported by several investigators, e.g. Carrol et al. (1980), Cruddace \& Dupree (1984), Vilhu \& Heise (1986), Vilhu et al. (1988), Tsuru et al. (1992), McGale et al. (1996) and recently by Choi \& Dotani (1998). The latter used the ROSAT Position Sensitive Proportional Counter to carry out a spectral survey of 7 X-ray bright W UMa contact systems. The total luminosities derived range from $4.4 \times 10^{29}$ to $3.3 \times 10^{30} \mathrm{erg} \mathrm{s}^{-1}$. The value of $\mathrm{X}$-ray luminosity of $\mathrm{BH}$ Cas is found inside this range.

Acknowledgements. The computations were performed at ACK "Cyfronet" in Cracow under grant No. KBN/UJ/015/95, which we gratefully acknowledge. This project was supported by the NATO fellowship No. DOO 902 98/99 to S. Zola and the NATO linkage grant No. CRG.LG.972172. We would like to thank J. Krzesinski for sharing his expertise in reducing CCD frames with DAOPHOT, W. Lu and S. Rucinski for obtaining the spectrum of BH Cas. Finally, we would like to acknowledge comments of an anonymous referee which significantly improved the presentation of this work.

\section{References}

Ahnert, P., \& Hoffmeister, C. 1943, Kleine Veroff. Univ. Berlin Babelsberg, 28, 4

Beljawsky, S. 1931, Astron. Nachr., 243, 115

Bessell, M. S. 1979, PASP, 91, 589

Brandt, W., Ward, M., Fabian, A., \& Hodge, P. 1997, MNRAS, 291, 709

Carroll, R. W., Cruddace, R. G., Friedman, H., et al. 1980, ApJ, 235, L77

Choi, C. S., \& Dotani, T. 1998, ApJ, 492, 761

Claret, A., Díaz-Cordovés, J., \& Gimenez, A. 1995, A\&AS, 114,247

Cruddace, R. G., \& Dupree, A. K. 1984, ApJ, 277, 263

Díaz-Cordovés, J., Claret, A., \& Gimenez, A. 1995, A\&AS, 110,329

Harmanec, P. 1988, Bull. Astron. Inst. Czech., 39, 329

Hilditch, R. W., King, D. J., \& McFarlane, T. M. 1988, MNRAS, 231, 341

Kreiner, J. M., Krzesinski, J., Pokrzywka, B., et al. 1993, Proc. of the IAU Coll. 136, ed. I. Elliot, \& C. J. Buttler, 80

Kukarkin, B. 1938, Veranderl. Sterne Nishni-Novgorod, 5, 195

Kwee, K. K., \& van Woerden, H. 1956, Bull. Astron. Inst. of Netherlands, 12, 327

Lu, W., \& Rucinski, S. M. 1999, AJ, 118, 515

Maceroni, C., \& van't Veer, F. 1996, A\&A, 311, 523

McGale, P. A., Pye, J. P., \& Hodgkin, S. T. 1996, MNRAS, 280,627

Metcalfe, T. S. 1994, IBVS, 4038

Metcalfe, T. S. 1999, AJ, 117, 2503

Mochnacki, S. W., \& Doughty, N. A. 1972a, MNRAS, 156, 51

Mochnacki, S. W., \& Doughty, N. A. 1972b, MNRAS, 156, 243

Niarchos, P. G., \& Duerbeck, H. W. 1991, A\&A, 247, 399

Niarchos, P. G., \& Duerbeck, H. W. 1997, Odessa Astronomical Publications, 10, 37

Priest, E., Parnell, C., \& Martin, S. 1994, ApJ, 427, 459

Sinachopoulos, D., Dapergolas, A., \& Kontizas, E. 1998, Memoirs of the National Observatory of Athens, Ser. I, No. 36

Stetson, P. B. 1987, PASP, 99, 191

Tsuru, T., Makishima, K., Ohashi, T., et al. 1992, MNRAS, 255,192

Vandenberg, D. A. 1985, ApJS, 58, 711

Van Hamme, W., \& Wilson, R. E. 1985, A\&A, 152, 25

Vilhu, O., \& Heise, J. 1986, ApJ, 311, 937

Vilhu, O., Caillault, J. P., \& Heise, J. 1988, ApJ, 330, 922

Wilson, R. E. 1978, ApJ, 234, 885

Wilson, R. E. 1979, ApJ, 234, 1054

Wilson, R. E. 1993, Documentation of Eclipsing Binary Computer Model

Wilson, R. E., \& Devinney, E. J. 1971, ApJ, 166, 605

Wilson, R. E., \& Devinney, E. J. 1973, ApJ, 182, 539 